\title{
A magnetic resonance imaging study of gastric motor function in patients with dyspepsia associated with Ehlers- Danlos Syndrome-Hypermobility Type: A feasibility study
}

Key points:

- "Aberrant gastric motility, accommodation, transit time likely play a role in dyspepsia. MRI has the ability to quantitatively evaluate these processes but remains underused due the length of existing gastric protocols and lack of a defined role in the clinical care pathway."[AM1]

- In this study we propose a $60 \mathrm{~min}$ protocol using a water stimulus which reveals differences between Ehlers Danlos patients with dyspepsia and healthy matched controls.

- This proposed MRI protocol is pragmatic and could take place in the clinical setting, offering multifactorial insights into gastric motor function.

\section{Abstract:}

Background: The clinical use of Magnetic Resonance Imaging (MRI) for investigating gastric motor function in dyspepsia is limited, largely due to protocol complexity, cost and limited availability. In this study we explore the feasibility of a sub 60-minute protocol using a water challenge to assess gastric emptying, motility and accommodation in a cohort of Ehlers Danlos Syndrome Hypermobility type (EDS-HT) patients presenting with dyspepsia.

Methods: 9 EDS-HT patients (mean age 33, range 26-50 all female) with a history of dyspepsia were recruited together with 9 matched controls. Subjects fasted for $6 \mathrm{~h}$ prior to MRI. A baseline anatomical and motility scan was performed after which the subjects ingested $300 \mathrm{~mL}$ water. The anatomical and motility scans were then repeated every 10 minutes to a total of $60 \mathrm{~min}$. Gastric emptying time, motility and accommodation were calculated based on the observations of two observers for each EDS-HT subject and compared to their matched control using paired statistics 
Key Results: Median motility increase following the water challenge was lower in EDS-HT subjects (11\%, range $0-22 \%)$ compared to controls (22\%, range 13 $56 \%), P=0.03$. Median gastric emptying time was non-significantly decreased in EDS-HT subjects (12.5min, range 6-27) compared to controls (20min, range 7$30), P=0.15$. Accommodation was non-significantly reduced in EDS-HT subjects (56\% increase, range 32\%-78\%) compared to healthy controls (67\% increase, range $52 \%-78 \%$ ), $\mathrm{P}=0.19$

Conclusions \& Inferences: This study demonstrates the feasibility of a water challenge MRI protocol to evaluate gastric physiology in the clinical setting. Motility differences between EDS-HT and controls are worthy of further investigation.

\section{Introduction:}

Dyspepsia is a gastrointestinal disorder characterized by upper abdominal discomfort or pain and symptoms of meal-related fullness or satiety, and has a significant impact on individuals' quality of life as well as health services [1]. Effective management is therefore an important clinical and societal imperative. Several mechanisms have been postulated to play a role in the pathogenesis of dyspepsia, including abnormalities in gastric motor and sensory function and, more recently, low-grade duodenal inflammation summarized recently by Stanghellini et al.[1]. Delayed gastric emptying, commonly referred to as gastroparesis, is found both in organic disorders such as diabetes and the more common functional dyspepsia (FD), estimated to have a prevalence of the $20 \%$ in the Western population[2]. Indeed, around one third of patients with functional dyspepsia have delayed gastric emptying, although correlation with symptoms is generally poor[3]. Gastric emptying is currently usually assessed with scintigraphy or $\left(\mathrm{C}^{13} / \mathrm{C}^{12}\right)$ breath testing which can provide some insight into aberrant physiology[4], [5].

It is clear however that gastric disorders are in fact underpinned by a spectrum of aetiologies, and manifest as a wide range of altered physiological process including abnormal gastric motility, accommodation, and response to nutrients. While a range of more specialized tests exist, for example barostat, their complexity, cost and discomfort for patients largely preclude use outside a research setting[6]. Therapeutic outcomes directed by relatively simplistic gastric empting studies are frequently poor and emptying studies themselves are weak endpoints in clinical trials of new therapeutic agents[7].

Alternatively, magnetic resonance imaging (MRI) provides sufficient spatial and temporal resolution to dynamically evaluate gastric physiology in real time, and measurement of emptying is already well validated[8]-[11]. Furthermore, recent advances in imaging analysis technologies now allow semi-automated quantitation of a range of novel gastric motility metrics. MRI is widely available, safe, increasingly cost effective, and employed widely in gastrointestinal conditions such as Crohn's Disease[12]-[14]. However clinical uptake in assessing gastric physiology is limited, in part due to the complexity of current 
protocols, which require a nutritionally pre-specified meal challenge and prolonged imaging acquisition, often taking up several hours of MRI scanner time. Thereafter, data analysis is complex and usually confined to specialist centres. There is a need to develop simple and effective gastric MRI protocols which capture the range of gastric physiology, but can be more easily be translated into the clinic.

The purpose of this study is to address current limitations of gastric MRI by proposing a relatively simple, well tolerated and time efficient protocol. Specifically we propose a sub 60min protocol to examine gastric motility, accommodation and emptying together with the duodenal response following a simple water stimulus.

We apply our protocol to an exemplar cohort of Ehlers Danlos syndrome hypermobility type (EDS-HT) patients with functional dyspepsia, a condition for which alterations in gastric motility have recently been described [15]-[19]. The primary aim of the study was to demonstrate feasibility of the technique in this patient cohort. Secondary exploratory aims were to test for differences in gastric physiology in comparison to matched healthy controls.

\section{Materials \& Methods}

\section{Subject selection}

\section{EDS-HT patients}

The study was approved by the hospital ethics committee and each participant gave informed consent

A total of 16 EDS-HT patients referred to a tertiary neurogastroenterology clinic in the period of October 2014-June 2015 were approached to participate. Patients were eligible if they had a history of nausea, pain or bloating and had been diagnosed with postprandial distress subtype Functional Dyspepsia according to Rome III criteria by their clinical team. Of those approached, five declined to take part and one subject was unable to stop their analgesic medication. A total of 9 EDS-HT patients were recruited: mean age 33 (range 26 to 50), all female, mean BMI 25 (range 20 to 35). Five of the EDS-HT patients had a diagnosis of Postural Orthostatic Tachycardia Syndrome (POTS), although a diagnosis of POTS was not required to be eligible.

A healthy control set with matched age ( \pm 12 months), sex and BMI $( \pm 5 \%)$ was recruited from the BLIND student and staff population for each EDS-HT patient. Health was defined as no history of gastrointestinal disease (including irritable 
bowel syndrome defined according to Rome III criteria), previous abdominal surgery and no current gastrointestinal symptoms (e.g. nausea, bloating, pain) suggestive of abnormal gastrointestinal function. Subjects were excluded if they had a contraindications to MRI.

\section{MRI Protocol}

\section{Subject preparation}

All EDS-HT patients temporarily stopped any motility influencing medication including laxatives, pro-kinetics for gastric emptying, opioid analgesics etc. for 5 days before undergoing MRI. Prior to MRI, all subjects fasted for 6 hours and avoided caffeinated drinks. Subjects were also instructed to avoid over the counter medications e.g. ibuprofen and smoking or drinking alcohol before the day of the scan.

\section{MRI protocol}

Subjects lay prone in a 3 Tesla Philips Achieva MRI scanner using the manufacturers torso coil. The scan protocol consisted of initial planning 'scout' sequences. The study coordinator (BLIND) supervised the positioning of the anatomical and motility sequences. The anatomical sequence was used to capture the total stomach volume for emptying and accommodation measures. The motility acquisition consisted of a rapid series of single slice acquisitions repeated in the coronal plane showing the greatest gastric volume, at a temporal resolution of 0.6 images per second over a period of 20 seconds. Sequence parameters for both are provided in Table 1 and performed on inspiration breath-hold.[AM2]

Following baseline acquisitions, a $300 \mathrm{~mL}$ tap water drink was given to the subject. Each subject drank the water through a straw while lying prone in the MRI bore with no more than 5 minutes between baseline and the first postchallenge scan. A schematic of the scan timings is presented in figure 1. Specifically, anatomical and motility sequence acquisitions took $5 \mathrm{~min}$ to acquire and were repeated 3 times, at 10 minute intervals. All subjects were able to consume the drink in the prone position and the total scan duration lasted no longer than 1 hour.

\section{Data analysis}

The stomach was manually segmented using the image viewing platform Osirix (Purview, Switzerland) by two independent readers blind to clinical details (BLIND, PhD student 2 years experience, BLIND, Gastroenterologist 4 years experience). In summary, the gastric volume (from lower oesophageal junction to pylorus) was segmented on each coronal slice using a polygonal ROI tool with each area multiplied by the slice thickness to create a total gastric volume. The 
gastric volumes were averaged across the two readers for the primary analysis described below.

\section{Gastric Emptying time}

Gastric emptying time was defined as the time taken for the stomach volume to decrease by $150 \mathrm{~mL}$ following ingestion of the water challenge adjusted by baseline volume (i.e. baseline volume plus $300 \mathrm{~mL}$ drink). Specifically, the gastric volume was measured immediately following the oral water challenge (baseline,) A gastric emptying curve was constructed using the 15min (baseline), $30 \mathrm{~min}$ and $45 \mathrm{~min}$ time points with the gastric empting time defined as time taken for half of the ingested water challenge to leave the stomach[9], [20].

\section{Gastric motility}

Time-series images of the stomach were processed with previously validated motility registration algorithm[21]. The algorithm is designed to quantify local deformation generated by the movement of the stomach wall. The deformation fields generated by the registration are used to provide a numerical surrogate for motility expressed as a unitless numerical value (standard deviation of the Jacobian determinant) that can be displayed as a motility colormap (Figure $2 \mathrm{~B}, \mathrm{E}, \mathrm{H}, \mathrm{K})$. The study coordinator performed the registration which automatically generated a single composite motility reference image (Figure $2 A, D, G, J)$ which was provided to the readers. Motility data before and immediately after the water challenge were used for the analysis.

Both readers manually segmented the stomach on the reference image only (Figure 2A,D,G,J) blind to motility information using a dedicated MATLAB software graphical user interface. The ROI coordinates were automatically applied by the study coordinator to the parametric motility map to obtain the gastric motility score for each subject averaged across the two readers.

\section{Gastric accommodation}

The gastric incisura was defined by an experienced consultant radiologist (BLIND) and annotated using a single line ROI on the baseline and immediate post water challenge datasets (two time points) (figure 3). This annotated data was passed to the two readers who manually and independently segmented the gastric volume above the level of the Incisura. The percentage change in aboveincisura volume (considered to correspond to fundic volume) was calculated between time point 1 and the post-drink volume 2. Accommodation was presented as the relative change in supra incisura gastric volume for each subject, averaged across the 2 readers.

\section{Duodenal response}


Two measures of duodenal function were measured for each subject. First, the duodenal volume, extending $10 \mathrm{~cm}$ beyond the pylorus, was calculated pre and at two time points ( 5 and 20 minutes) after the water challenge. Segmentation was performed by a consultant gastroenterologist (BLIND) using the same sequences as the gastric motility calculation. Second, the dynamic data was assessed to explore changes in duodenal motility at two time points, pre and immediately post water challenge.

\section{Statistical analysis}

All data was considered as paired between EDS-HT patients and their age, sex and BMI matched control. Data distribution was assessed using the Shapiro-Wilk test $<0.05$ and non-parametric tests were used where data could not be normalised.

Inter-reader agreement was assessed between the two readers for the motility scores and gastric volumes using Bland Altman statistics with adjustment for clustered data, and an intra-class correlation (ICC) statistic.

Gastric emptying times between EDS-HT subjects and controls were compared using Wilcoxon signed rank test (non-parametric paired T-test).

Gastric motility before and after the water bolus was assessed using Wilcoxon signed rank test. The relative change in motility after the water bolus (expressed as \% change compared to baseline) was compared between EDS-HT patients and controls by Mann-Whitney U test.

Gastric accommodation between EDS-HT subjects and their controls was compared using Wilcoxon signed rank test. The relationship between accommodation and 1) emptying and 2) motility was explored through Spearman's correlation.

Duodenal distension (based on changes in volume) was calculated across the three time points and compared within subject using Wilcoxon signed rank between EDS-HT patients and healthy controls using the Mann Whitney U test.

Duodenal motility before and after the water bolus was assessed within subjects using Wilcoxon signed rank test and between the EDS-HT patients and healthy controls using the Mann Whitney U test 


\section{Results}

All 18 subjects recruited were able to comply fully with the protocol and complete the study.

\section{Inter-reader agreement}

Gastric volumes from each subject time point were calculated (3 volumes for each subject). Agreement achieved an ICC of 0.96. Bland-Altman analysis showed a mean difference of $-7 \mathrm{~cm}^{3}, 95 \%$ limits of agreement at $82 \mathrm{~cm}^{3}$ across a range of 26 to $567 \mathrm{~cm}^{3}$ (figure $4 \mathrm{~A}, \mathrm{~B}$ ).

Gastric motility score was calculated at 2 time points for each subject (36 in total). The ICC was 0.99 , and BA LoA showed a mean difference of $<0.001$ units, $95 \%$ limits of agreement at 0.012 units across a range of 0.085 to 0.29 units (figure 4C,D).

\section{Gastric emptying}

According to the a priori definition, the mean gastric emptying time for EDS-HT patients was 12.5min (range 6 to 27, with one apparent outlier) Figure 5. The mean emptying time in controls was $20 \mathrm{~min}$ (range 7 to $30 \mathrm{~min}$ ). The mean difference in emptying time was 7.5 minutes which was not statistically significant $\mathrm{P}=0.15$.

Repeating the analysis, this time excluding the outlier, produced a significant result $\mathrm{P}=0.032$. Of note, the outlier had a BMI of 35 .

\section{Gastric motility}

The mean baseline motility across all subjects was 0.14 units (range 0.075 to 0.25 ) and following water was 0.17 (range 0.075 to 0.28 ) with a statistically significant difference of 0.03 units $\mathrm{P}=0.005$ (figure 6a).

EDS-HT patients had a mean $11 \%$ (range 0 to $22 \%$ ) increase in motility while the controls had a mean $22 \%$ increase (range 13 to $56 \%$ ). The difference of $11 \%$ was statistically significant $(p=0.03$ (figure $6 b$ ).

\section{Gastric accommodation}

The mean accommodation increase in EDS-HT subjects was $56 \%$ (range $32 \%$ to $78 \%$ ) and in healthy controls $67 \%$ (range $52 \%$ to $78 \%$ ). The difference of $11 \%$ was not statistically significant, $\mathrm{P}=0.19$ (figure 7/Or appendix 1)

A significant positive correlation was seen between accommodation and gastric emptying time, $\mathrm{R}=0.52$, $\mathrm{P} 0.04$ (figure $7 \mathrm{~b}$ ). There was no significant correlation between accommodation and motility, $\mathrm{R}=0.1, \mathrm{P}=0.86$. 


\section{Duodenal response}

The mean duodenal volumes across the three time points for the EDS-HT subjects pre water challenge was $22.0 \mathrm{~cm}^{3}\left(4\right.$ to $\left.43 \mathrm{~cm}^{3}\right)$, immediately after water challenge was $35.7 \mathrm{~cm}^{3}$ (range 8 to $69 \mathrm{~cm}^{3}$ ) and $42.1 \mathrm{~cm}^{3}$ (range 5 to $20 \mathrm{~cm}^{3}$ ) after the challenge. In the healthy controls, the mean volume was $22.2 \mathrm{~cm}^{3}$ (range 1 to $53 \mathrm{~cm}^{3}$ ), $34.1 \mathrm{~cm}^{3}$ (range 10 to $66 \mathrm{~cm}^{3}$ ) and $30.8\left(\right.$ range 10 to $60 \mathrm{~cm}^{3}$ ) respectively across the three time points (Figure 8A/Or appendix 2).

There was a significant increase in duodenal volume between baseline and post challenge volumes at both time points $(\mathrm{P}<0.001$ and $\mathrm{P}=0.003$ respectively).

Duodenal motility data from two EDS-HT subjects and three controls could not be analysed due to low data quality (duodenum could not be visualised on dynamic sequences). The pre water challenge mean duodenal motility in the EDS-HT cohort was 0.19 (range 0.08 to 0.38 ) rising to 0.23 (range 0.07 to 0.4 ) post challenge. In the healthy controls, the mean motility was 0.23 (range 0.14 to 0.44 ) and 0.27 (range 0.15 to 0.51 ) respectively (figure 8b/Or appendix 2).

No significant difference was observed between the EDS-HT and control subjects. 


\section{Discussion}

This feasibility study explored the ability of MRI to evaluate gastric emptying, motility and accommodation using a simple water challenge protocol. The technique was applied to a cohort of EDS-HT patients and healthy, matched controls. The EDS-HT patients demonstrated significantly reduced motility compared to controls. The protocol was rapid, well tolerated and, while the number of patients was small, supports the use of MRI to interrogate multiple aspects of gastric physiology simultaneously which might be relevant for patients with functional and organic disorders of gastric function.

A difference in post water challenge motility between EDS-HT patients and controls was the main statistically significant finding in this study. Gastric motility can be easily visualised by capturing a time-series of coronal slice through stomach[4]. Although widely reported in the literature, a standardised method for calculating and evaluating gastric motility has yet to be defined [10]. We applied an image registration based approach to calculate a motility map which is based on the analysis of the registration deformation field. This approach has been successfully applied in the small bowel[21] and colon[22]. The output is a surrogate measure of motility, but has been shown to be reproducible and has proven clinical utility, particularly in the small bowel [23]. Segmentation of the stomach by the observer takes around 30 seconds only. Furthermore, the visual output of a colour motility map is intuitively simple to understand [23], [24]. Importantly, in the current study there was very high inter reader agreement, suggesting the technique is reproducible between observers. We also demonstrated a significant increase in motility following the simple water challenge suggesting that distension alone (without calories) is sufficient to generate contractions, an observation in agreement with previous observations by Teramato et al[25].

We further present a method for calculating gastric accommodation based on assessing the above-incisura volume change following the oral challenge. The concept behind the measure was to act as a surrogate for the expansive capacity in the body of the stomach, expressed as a simple, relative change in volume. Although we found no statistically significant difference between EDS-HT patients and controls, we believe the measure may play a useful role in understanding aberrant gastric physiology. The correlation between our measure of accommodation and emptying time gives additional insight into the functional importance of accommodation in gastric dynamics and suggests measurement may provide important information in functional disease.

Measurement of gastric emptying using MRI has been extensively validated [11], [26]-[29] and previously descrbed using a water stimulus[30]. Based on this methodology, we believe our observation that EDS-HT subjects showed increased gastric emptying times is likely a real phenomenon although further investigations are necessary to make a definitive statement with regards to EDSHT. Of note, the difference in gastric emptying times between EDS-HT patients and controls was only statistically significant after post hoc removal of an 
apparent outlier (with a BMI of 35). This, combined with our small cohort, means caution must be applied to the finding and clearly larger studies are indicated.

A substantial literature exists on the influence of different meals on gastric emptying times[31]-[33]. Use of a simple water-challenge to provoke a physiological response in the stomach could therefore be seen a limitation in this study. Although we recognise the benefits of a validated test meal, a simple water challenge has several advantages within the context of exploring gastric physiology in routine clinical practice and builds on early prelminiary work elsewhere[25], [30]. Firstly, the total scan duration can be greatly decreased with a total investigation lasting approximately 30 minutes (although in this initial study we scanned for longer). A nutritionally balanced test meal by comparison may take several hours to clear the stomach[34] and creates logistical problems with respect to patient throughput on MRI platforms. Regardless of the mechanism of effect, the efficacy of water to provoke motility appears to be a real phenomenon supported by the findings of Teramoto et al who also used water to stimulate gastric motility - one of the only other studies in the literature, to our knowledge, to describe this response[25]. Furthermore, the water drink can be easily consumed in the scanner with minimal risk to the patient and is well tolerated and can be easily prepared by the radiographer

We were able generate data on both duodenal volume and motility in most patients to further promote the extra-gastric versatility of this approach. Recent data suggests an aberrant duodenal response by underpin certain abnormalities in gastric motor and sensory function[35], [36]. Using our technique, we were able to demonstrate the expected rise in duodenal volume following the water bolus and also saw changes in duodenal motility, although these did not seem to differ between EDS-HT patients and controls.

As a feasibility study, our primary aim was to test the practicality and performance of our approach in assessing several aspects of gastric physiology. We chose to study a cohort of EDS-HT patients as it has been demonstrated that patients with functional GI disorders, most notably functional dyspepsia commonly meet the criteria for EDS-HT-HT, [15]-[18]. Several hypotheses have been formulated with regards to the potential aetiology of dyspeptic symptoms in this cohort [37]. One hypothesis suggests that EDS-HT patients are characterized by connective tissue anomalies which may underlie alterations in gastric motility [16]. There is a strong clinical need for a test to better phenotype gastric physiology in this patient group in whom the differentiation between organic and functional disorders is difficult. Our data, albeit part of a pilot study, does support there being underlying abnormal gastric motility in EDS-HT patients with upper GI symptoms.

A limitation of our study is that although all recruited EDS-HT patients presented clinically with symptoms compatible with functional dyspepsia ,we did not apply validated questionnaires to document the severity of the symptoms. Going forward, there is a clear need to fully characterise recruited cohorts using currently available tools to better understand the observations made using MRI. 
As noted above our cohorts were small but reasonable for a pilot study of this nature

In summary, MRI remains a promising technique for persuing a multidimensional understanding of gastric physiology. Our proposed MRI technique is simple to perform, objective and practical with excellent inter-observer agreement. Our data also supports to need for larger studies in the EDS-HT patient cohort to understand the mechanisms underpinning dyspeptic symptoms 


\section{References}

[1] V. Stanghellini, F. K. L. Chan, W. L. Hasler, J. R. Malagelada, H. Suzuki, J. Tack, and N. J. Talley, "Gastroduodenal Disorders," Gastroenterology, vol. 150, no. 6, pp. 1380-1392, May 2016.

[2] J. Tack, N. J. Talley, M. Camilleri, G. Holtmann, P. Hu, J.-R. Malagelada, and V. Stanghellini, "Functional Gastroduodenal Disorders," Gastroenterology, vol. 130, no. 5, pp. 1466-1479, 2006.

[3] V. Stanghellini and J. Tack, "Gastroparesis: separate entity or just a part of dyspepsia?," Gut, vol. 63, no. 12, pp. 1972-8, Dec. 2014.

[4] A. E. Bharucha, M. Camilleri, E. Veil, D. Burton, and A. R. Zinsmeister, "Comprehensive assessment of gastric emptying with a stable isotope breath test.," Neurogastroenterol. Motil., vol. 25, no. 1, pp. e60-9, Jan. 2013.

[5] G. Tougas, E. Y. Eaker, T. L. Abell, H. Abrahamsson, M. Boivin, J. Chen, M. P. Hocking, E. M. M. Quigley, K. L. Koch, A. Z. Tokayer, V. Stanghellini, Y. Chen, J. D. Huizinga, J. Ryden, I. Bourgeois, and R. W. McCallum, "Assessment of gastric emptying using a low fat meal: establishment of international control values," Am. J. Gastroenterol., vol. 95, no. 6, pp. 1456-1462, Jun. 2000.

[6] D. Ang, "Measurement of gastric accommodation: a reappraisal of conventional and emerging modalities.," Neurogastroenterol. Motil., vol. 23, no. 4, pp. 287-91, Apr. 2011.

[7] P. J. Pasricha and W. Snape, "Toward a Better Drug for Gastroparesis: The Problem With a Moving Target," Gastroenterology, vol. 151, no. 1. pp. 2022, 2016.

[8] A. E. Bharucha, R. A. Karwoski, J. Fidler, D. R. Holmes, R. A. Robb, S. J. Riederer, and A. R. Zinsmeister, "Comparison of manual and semiautomated techniques for analyzing gastric volumes with MRI in humans," AJP Gastrointest. Liver Physiol., vol. 307, no. 5, pp. G582-G587, Sep. 2014.

[9] H. Fruehauf, D. Menne, M. A. Kwiatek, Z. Forras-Kaufman, E. Kaufman, O. Goetze, M. Fried, W. Schwizer, and M. Fox, "Inter-observer reproducibility and analysis of gastric volume measurements and gastric emptying assessed with magnetic resonance imaging.," Neurogastroenterol. Motil., vol. 23, no. 9, pp. 854-61, Sep. 2011.

[10] L. A. Szarka and M. Camilleri, "Methods for measurement of gastric motility," AJP Gastrointest. Liver Physiol., vol. 296, no. 3, pp. G461-G475, Jan. 2009.

[11] C. Feinle, P. Kunz, P. Boesiger, M. Fried, and W. Schwizer, "Scintigraphic validation of a magnetic resonance imaging method to study gastric emptying of a solid meal in humans.," Gut, vol. 44, no. 1, pp. 106-11, Jan. 1999.

[12] J. Panes, Y. Bouhnik, W. Reinisch, J. Stoker, S. A. Taylor, D. C. Baumgart, S. Danese, S. Halligan, B. Marincek, C. Matos, L. Peyrin-Biroulet, J. Rimola, G. Rogler, G. van Assche, S. Ardizzone, A. Ba-Ssalamah, M. A. Bali, D. Bellini, L. Biancone, F. Castiglione, R. Ehehalt, R. Grassi, T. Kucharzik, F. Maccioni, G. Maconi, F. Magro, J. Martín-Comín, G. Morana, D. Pendsé, S. Sebastian, A. Signore, D. Tolan, J. A. Tielbeek, D. Weishaupt, B. Wiarda, and A. Laghi, "Imaging techniques for assessment of inflammatory bowel disease: joint 
ECCO and ESGAR evidence-based consensus guidelines.," J. Crohns. Colitis, vol. 7, no. 7, pp. 556-85, Aug. 2013.

[13] M. J. Steward, S. Punwani, I. Proctor, Y. Adjei-Gyamfi, F. Chatterjee, S. Bloom, M. Novelli, S. Halligan, M. Rodriguez-Justo, and S. A. Taylor, "Nonperforating small bowel Crohn's disease assessed by MRI enterography: derivation and histopathological validation of an MR-based activity index.," Eur. J. Radiol., vol. 81, no. 9, pp. 2080-8, Sep. 2012.

[14] K. Horsthuis, S. Bipat, P. C. F. Stokkers, and J. Stoker, "Magnetic resonance imaging for evaluation of disease activity in Crohn's disease: a systematic review.," Eur. Radiol., vol. 19, no. 6, pp. 1450-60, Jun. 2009.

[15] M. Castori, F. Camerota, C. Celletti, C. Danese, V. Santilli, V. M. Saraceni, and P. Grammatico, "Natural history and manifestations of the hypermobility type Ehlers-Danlos syndrome: a pilot study on 21 patients.," Am. J. Med. Genet. A, vol. 152A, no. 3, pp. 556-64, Mar. 2010.

[16] A. D. Nelson, M. A. Mouchli, N. Valentin, D. Deyle, P. Pichurin, A. Acosta, and M. Camilleri, "Ehlers Danlos syndrome and gastrointestinal manifestations: a 20-year experience at Mayo Clinic.," Neurogastroenterol. Motil., vol. 27, no. 11, pp. 1657-66, Nov. 2015.

[17] J.-D. Zeitoun, J. H. Lefèvre, V. de Parades, C. Séjourné, I. Sobhani, B. Coffin, and C. Hamonet, "Functional digestive symptoms and quality of life in patients with Ehlers-Danlos syndromes: results of a national cohort study on 134 patients.," PLoS One, vol. 8, no. 11, p. e80321, 2013.

[18] N. Zarate, A. D. Farmer, R. Grahame, S. D. Mohammed, C. H. Knowles, S. M. Scott, and Q. Aziz, "Unexplained gastrointestinal symptoms and joint hypermobility: is connective tissue the missing link?," Neurogastroenterol. Motil., vol. 22, no. 3, pp. 252-e78, Mar. 2010.

[19] A. Fikree, R. Grahame, R. Aktar, A. D. Farmer, A. J. Hakim, J. K. Morris, C. H. Knowles, and Q. Aziz, "A prospective evaluation of undiagnosed joint hypermobility syndrome in patients with gastrointestinal symptoms.," Clin. Gastroenterol. Hepatol., vol. 12, no. 10, pp. 1680-87.e2, Oct. 2014.

[20] H. L. Parker, C. L. Hoad, N. Hudders, C. Costigan, L. Marciani, E. Cox, P. A. Gowland, and M. R. Fox, "Mo1164 Validation of a Novel, Non-Invasive Assessment of Gastric Function and Gastric Emptying (GE) After a Large Liquid Nutrient Meal by Magnetic Resonance Imaging (MRI)," Gastroenterology, vol. 142, no. 5, p. S-610, May 2012.

[21] F. Odille, A. Menys, A. Ahmed, S. Punwani, S. A. Taylor, and D. Atkinson, "Quantitative assessment of small bowel motility by nonrigid registration of dynamic MR images.," Magn. Reson. Med., vol. 68, no. 3, pp. 783-93, Sep. 2012.

[22] A. Menys, V. Hamy, J. Makanyanga, C. Hoad, P. Gowland, F. Odille, S. A. Taylor, and D. Atkinson, "Dual registration of abdominal motion for motility assessment in free-breathing data sets acquired using dynamic MRI.," Phys. Med. Biol., vol. 59, no. 16, pp. 4603-19, Aug. 2014.

[23] A. Menys, S. A. Taylor, A. Emmanuel, A. Ahmed, A. A. Plumb, F. Odille, A. Alam, S. Halligan, and D. Atkinson, "Global Small Bowel Motility: Assessment with Dynamic MR Imaging," Nov. 2013.

[24] C. L. Hoad, A. Menys, K. Garsed, L. Marciani, V. Hamy, K. Murray, C. Costigan, D. Atkinson, G. Major, R. C. Spiller, S. A. Taylor, and P. A. Gowland, "Colon wall motility: comparison of novel quantitative semi-automatic 
measurements using cine MRI.," Neurogastroenterol. Motil., Nov. 2015.

[25] H. Teramoto, T. Shimizu, H. Yogo, Y. Nishimiya, S. Hori, T. Kosugi, and S. Nakayama, "Assessment of gastric emptying and duodenal motility upon ingestion of a liquid meal using rapid magnetic resonance imaging," Exp. Physiol., vol. 97, no. 4, pp. 516-524, Apr. 2012.

[26] A. Steingoetter, M. Fox, R. Treier, D. Weishaupt, B. Marincek, P. Boesiger, M. Fried, and W. Schwizer, "Effects of posture on the physiology of gastric emptying: A magnetic resonance imaging study," http://dx.doi.org/10.1080/00365520600610451, 2009.

[27] J. Fidler, A. E. Bharucha, M. Camilleri, J. Camp, D. Burton, R. Grimm, S. J. Riederer, R. A. Robb, and A. R. Zinsmeister, "Application of magnetic resonance imaging to measure fasting and postprandial volumes in humans.," Neurogastroenterol. Motil., vol. 21, no. 1, pp. 42-51, Jan. 2009.

[28] H. Fruehauf, A. Steingoetter, M. R. Fox, M. A. Kwiatek, P. Boesiger, W. Schwizer, M. Fried, M. Thumshirn, and 0. Goetze, "Characterization of gastric volume responses and liquid emptying in functional dyspepsia and health by MRI or barostat and simultaneous C-acetate breath test.," Neurogastroenterol. Motil., vol. 21, no. 7, pp. 697-e37, Jul. 2009.

[29] C. L. Hoad, H. Parker, N. Hudders, C. Costigan, E. F. Cox, A. C. Perkins, P. E. Blackshaw, L. Marciani, R. C. Spiller, M. R. Fox, and P. A. Gowland, "Measurement of gastric meal and secretion volumes using magnetic resonance imaging.," Phys. Med. Biol., vol. 60, no. 3, pp. 1367-83, Feb. 2015.

[30] D. M. Mudie, K. Murray, C. L. Hoad, S. E. Pritchard, M. C. Garnett, G. L. Amidon, P. A. Gowland, R. C. Spiller, G. E. Amidon, and L. Marciani, "Quantification of Gastrointestinal Liquid Volumes and Distribution Following a $240 \mathrm{~mL}$ Dose of Water in the Fasted State," Mol. Pharm., vol. 11, no. 9, pp. 3039-3047, Sep. 2014.

[31] L. Marciani, R. Faulks, M. S. J. Wickham, D. Bush, B. Pick, J. Wright, E. F. Cox, A. Fillery-Travis, P. A. Gowland, and R. C. Spiller, "Effect of intragastric acid stability of fat emulsions on gastric emptying, plasma lipid profile and postprandial satiety," Br. J. Nutr., vol. 101, no. 06, p. 919, Mar. 2009.

[32] P. J. Collins, M. Horowitz, and B. E. Chatterton, "Proximal, distal and total stomach emptying of a digestible solid meal in normal subjects.," $B r . J$. Radiol., vol. 61, no. 721, pp. 12-8, Jan. 1988.

[33] M. Edelbroek, M. Horowitz, A. Maddox, and J. Bellen, "Gastric emptying and intragastric distribution of oil in the presence of a liquid or a solid meal.," $J$. Nucl. Med., vol. 33, no. 7, pp. 1283-90, Jul. 1992.

[34] L. Marciani, E. F. Cox, C. L. Hoad, S. Pritchard, J. J. Totman, S. Foley, A. Mistry, S. Evans, P. A. Gowland, and R. C. Spiller, "Postprandial Changes in Small Bowel Water Content in Healthy Subjects and Patients With Irritable Bowel Syndrome," Gastroenterology, vol. 138, no. 2, pp. 469-477.e1, 2010.

[35] l. kloetzer, w. d. chey, r. w. mccallum, k. l. koch, j. m. wo, m. sitrin, l. a. katz, j. m. lackner, h. p. parkman, g. e. wilding, j. r. semler, w. l. hasler, and b. kuo, "Motility of the antroduodenum in healthy and gastroparetics characterized by wireless motility capsule," Neurogastroenterol. Motil., vol. 22, no. 5, pp. 527-33, e117, Jan. 2010.

[36] K. Barshop, K. Staller, J. Semler, and B. Kuo, "Duodenal rather than antral motility contractile parameters correlate with symptom severity in gastroparesis patients," Neurogastroenterol. Motil., vol. 27, no. 3, pp. 339- 
346, Mar. 2015.

[37] N. J. Talley, "Functional gastrointestinal disorders as a public health problem.," Neurogastroenterol. Motil., vol. 20 Suppl 1, pp. 121-9, May 2008.

\section{Figure text}

Figure 1. Schematic of MRI scan timings. Planning represents positioning the sequence to cover the stomach. Imaging included the anatomical and motility sequences. The water challenge was provided after the baseline imaging while the patient lay in the scanner. [AM3]

Figure 2. Healthy control (A-F) and EDS-HT subject (G-L). Baseline and post water challenge reference images in healthy $(A, D)$ and EDS-HT $(G, H)$ subjects. Corresponding motility maps (Healthy: B,E and EDS-HT: H,K) and fused images (Healthy: C,D and EDS-HT: I,L). A region of interest (ROI) was used by both observers to delineate the stomach blind to motility data (Figures A,D,G,J only). An increase in gastric motility, particularly at the antrum can be seen following challenge in the healthy control (red arrow).

Figure 3. Gastric accommodation measure with the incisura represented by a dotted red line. The above incisura volume was measured at baseline $(A)$ and post water (B) challenge. The Incisura was identified and delineated by an expreicnd consultant radiologist with manual segmenentation then peformed, independently by two readers of the upper and lower portions of the stomach. [AM4]

Figure 4. Results of inter-reader agreement of gastric volumes (A,B) and motility (C,D) assessed with Intra-Class Correlation coefficient and Bland-Altman Limits of Agreement respectively. Overall, good agreement was observed without evident bias in either of the readers observations. [AM5]

Figure 5. Boxplots to demonstrate gastric emptying time in EDS-HT and controls. The red cross signifies an outliying datapoint (this subject had a BMI of 35). [AM6]

Figure 6. All subjects motility pre/post water challenge (A) with healthy controls indicated with a green dotted line and EDS-HT patients with a black dotted line. The relative change in motility pre and post water challenge as a percentage presented as a boxplot (B).[AM7]

Figure 7. Relative change in volume pre/post water as a percentage presented as a boxplot (A) and correlation of accommodation against emptying time for all subjects displaing a significant positive relationship [AM8](B).

Figure 8. Duodenal volumes at three time points pre (T1) water challenge, immediately after (T2) and approximately 20 minutes after water challenge (T3) 
(A). Duodenal motility pre and post water challenge (B). Green lines represent healthy controls, black lines are EDS-HT subjects.

\section{Table Text}

Table 1. Details of anatomical and motility scan sequences. 